\section{Abatacept inhibits immune response but does not adversely affect response to vaccination}

Abatacept, a CTLA4-Fc fusion protein that modulates T-cell activation, has been developed for the treatment of rheumatoid arthritis. Tay et al. evaluated the effect of abatacept on vaccination with tetanus toxoid vaccine and 23-valent pneumococcal vaccine in 80 healthy individuals. Volunteers were vaccinated 2 weeks before, 2 weeks after, or 8 weeks after abatacept administration. A control group did not receive abatacept.

Abatacept reduced the geometric mean titers for both vaccines. The greatest effect was seen in those who were vaccinated 2 weeks after abatacept administration. By contrast, abatacept produced only a small decrease in antibody levels in those individuals who were vaccinated before administration. At least $60 \%$ of all those who received abatacept responded (defined as a $\geq 2$-fold increase in antibody titer) to tetanus vaccination compared with $75 \%$ of the control group, and more than $70 \%$ of treated individuals responded to at least three serotypes of pneumococcal vaccine compared with $100 \%$ of the control group. The most common adverse effects in the treated groups were headache, injection-site pain and viral infection. One serious adverse event of generalized urticaria was reported after abatacept administration.

The authors conclude that although abatacept has an inhibitory effect on the immune response, it does not prevent healthy individuals from developing a satisfactory response to vaccination with tetanus toxoid or pneumococcal vaccines. Studies evaluating the vaccination response in patients with rheumatoid arthritis are ongoing.

Original article Tay L et al. (2007) Vaccination response to tetanus toxoid and 23-valent pneumococcal vaccines following administration of a single dose of abatacept: a randomized, open-label, parallel group study in healthy subjects. Arthritis Res Ther [doi: 10.1186/ar2174]

\section{TNF promoter polymorphisms influence the outcome of RA}

Raised TNF concentrations have been implicated in the pathophysiology of rheumatoid arthritis (RA), and genetic factors are thought to influence the circulating levels of TNF. Fonseca et al. analyzed the promoter area of the TNF gene (where the highest genetic variability is thought to be concentrated) between the nucleotides -1318 and +49 to assess whether clinical activity and severity of RA are influenced by TNF promoter markers.

The study included 491 patients (mean age $57 \pm 13.3$ years) who had a mean RA disease duration of $12.7 \pm 10.5$ years. RA activity was assessed by the Disease Activity Score and functional ability by the Health Assessment Questionnaire. Systemic manifestations, radiological damage, use of disease-modifying antirheumatic drugs, previous joint surgeries, and work disability were also assessed. Ten single nucleotide polymorphisms (SNPs) were genotyped, but five were excluded because they did not fall within the Hardy-Weinberg equilibrium or were monomorphisms.

SNPs at $-238,-308$, and -857 were associated with systemic manifestations, radiological damage, work disability and a need for joint surgery. A weak association was seen between SNPs at -863 and -238 and high Disease Activity Score, poor Health Assessment Questionnaire result and raised rheumatoid factor concentration; these associations varied with disease duration. In patients with $>10$ years of disease duration, an association was found between haplotypes and radiological progression.

The authors suggest a prospective study is needed to validate their findings and to determine the value of genotyping TNF promoter sequences in the assessment of the progression of RA.

Original article Fonseca JE et al. (2007) Contribution for new genetic markers of rheumatoid arthritis activity and severity: sequencing of the tumour necrosis factor alpha gene promoter. Arthritis Res Ther 9: R37

\section{Gabapentin reduces pain severity in patients with fibromyalgia}

Gabapentin, a structural analog of the neurotransmitter GABA, has antinociceptive properties in patients with hypersensitized pain conditions. Arnold and colleagues, therefore, assessed the safety and efficacy of gabapentin in patients with fibromyalgia.

This randomized, double-blind, placebocontrolled trial included patients who met the American College of Rheumatology criteria for 